\title{
PRESSURE MEASUREMENT FOR VACUUM MOTOR IN AUTOMATED TELLER MACHINE DISPENSER
}

\author{
S.R Jayalogainiyan ${ }^{1}$, K.S Srijan ${ }^{2}$, A. Vijayalakshmi ${ }^{3}$ \\ ${ }^{1}$ PG Scholar, ECE Department, Sri Manakula Vinayagar Engineering College, Puducherry, India \\ ${ }^{2} P G$ Scholar, ECE Department, Sri Manakula Vinayagar Engineering College, Puducherry, India \\ ${ }^{3}$ Associate Professor, ECE Department, Sri Manakula Vinayagar Engineering College, Puducherry, India
}

\begin{abstract}
Pressure measurement for vacuum motor in dispenser unit is a process to find the pressure generated by the vacuum motor present inside the dispenser unit of an Automated Teller Machine (ATM). The dispenser unit is used to load the cash deposited in the ATM machine, a bipolar stepper motor is used as a vacuum motor which is present inside dispenser is used to pick the currency by applying an amount of pressure and withdraw the cash through the cash disburser. The pressure generated by the vacuum motor to pick cash should be equal to the threshold value prescribed by the ATM manufacturer, variation in the pressure may effect in failure of picking cash or incorrect count of cash withdrawal. So this paper concentrates on measuring, displaying and storing the pressure values of vacuum motor present in ATM dispenser. The pressure is measured using pressure flow sensor and exhibited using Liquid Crystal Display (LCD). The ATMEGA-8 microcontroller is interfaced with the computer using USB serial communication and pressure value obtained is displayed using datalogger software.
\end{abstract}

Keywords- Automated Teller Machine (ATM), datalogger, dispenser, Liquid Crystal Display (LCD), threshold pressure, vacuum motor.

\section{INTRODUCTION}

An Automated Teller Machine is electronic device that delivers cash and performs additional financial transaction services on insertion of a magnetic coded card by the customer. Automated Teller Machine is also called Automated Banking Machine in some parts of the world it is also known as cash point, cash line, cash machine and Point of Sale machine etc. The innovation of modern innovation has made likely for their clients to interact and carry out banking facility with Automated Teller Machine and helps to receive cash directly from the machine or make deposit including checks without assistance of human being. Automated Teller Machine is a part of electronic banking [1] and a service offered by several banking sector to their customers. One of the main reason for banks to install Automated Teller Machine is rising cost of operating bank branches in several places. This made a rapid popularity because of customer's convenience. The details of existing Automated Teller Machine are discussed in section 2, and implementation of proposed system is described in section 3. The performance and result are discussed in section 4 and section 5 and 6 gives the conclusion and future enhancement respectively.

\section{AUTOMATED TELLER MACHINE}

An Automated Teller Machine is a real time terminal opted for the banking customer service with the support of central bank server and a centralized account database [2]. Automated Teller Machine provides currency withdraw and account balance management services. The block diagram of an ATM shown in Fig. 1 contains a processor, peripheral devices like card reader, monitor, keypad, bill storage and disburser unit, receipt printer. ATM is also comprised of system clock and remote account database [5] to transfer information with the bank's server.

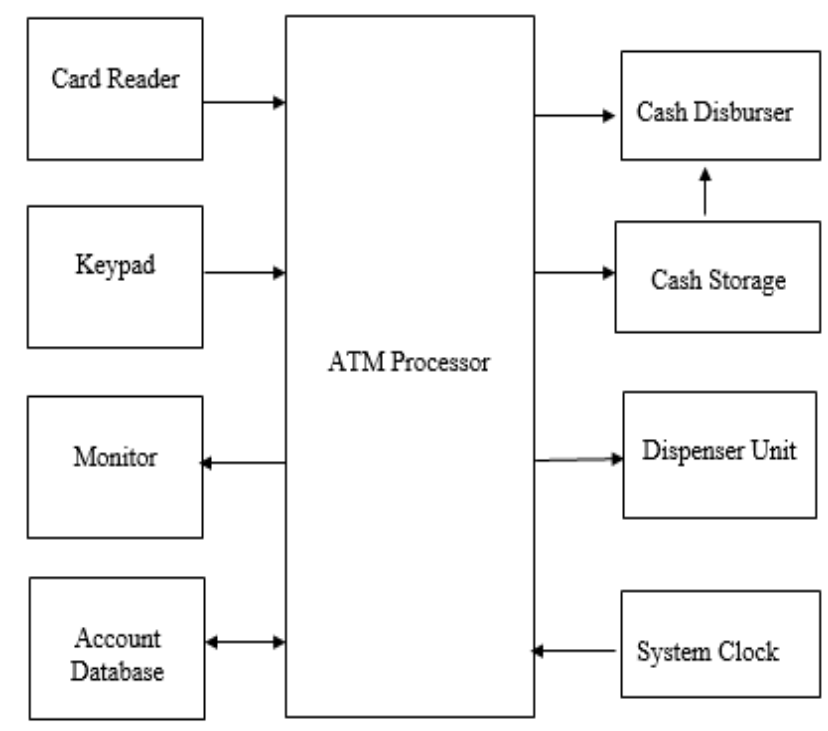

Fig. 1 Block diagram of ATM system

\subsection{ATM Card Reader}

The card reader acts as an interface device, which accepts an inserted valid bank card which is protected with PIN, will scan the predefined information on the card [10], and returns 
the card to the users. ATM card is comprised of the magnetic chip encoded [6] with user information along with the card number and validity of the card. Card reader is able to evaluate the information printed in the card [8] and the account details of the user and sends a request to the banks account database to process the transaction.

\subsection{ATM Keypad}

The keypad of an ATM is called as Encrypted Pin Pad (EPP) is an interface device [4] for banking customers entering vital information like personal identification number (PIN) and to process the essential data's in ATM. The input field of the PIN is represented as ( $\leq 4$ digits) entered from the keypad.

\subsection{ATM Monitor}

The monitor of the ATM system is an external device that displays system process and status information to the users. The output information represents a string letters $(\leq 255$ characters) by which it can be viewed in the monitor.

\subsection{Cash Storage}

The cash storage of the ATM system is the internal device that stores cash in different notes, which can be directed to the bills disburser in various combination [7]. The currency storage section contains the cassette which helps to store the cash built on the variance of the amount.

\subsection{Cash Disburser}

The cash disburser is an output device that delivers cash of requested amount from the cash storage to the customer. The field of amount disbursed is a system response signal of bills disbursed to the customer in the current transaction.

\subsection{ATM System Clock}

The system clock of the ATM is a model for event timing, process time management and system synchronization. The system clock affords an complete calendar clock with current time in the format HH:MM:SS as the time mentioning of the total system and a relative clock as a standard counter [7] of the ATM.

\subsection{ATM System Database}

The ATM system database is an internal centralized databank located in the bank's server. The system database scans the card details and the PIN for the authentication of the customer to verify the card validity and information logged in the particular account [5] such as the card holder, current balance and withdraw constraints.

\subsection{Dispenser Unit}

Cash dispenser contains currency cassettes which used to transport currency movement in the dispenser shown in Fig.2. The cash cassette is secured by providing seal on the lid latch and the reject currency is reverted to the elimination bin in the dispenser [9]. The elimination bin and the currency cassettes remain sealed and secured when ATM is opened. The currency in the cassettes moves under the pressure generated by the vacuum motor which helps to stack the currency in position [3]. The pick line has eccentric movement which inclines to drive the currency stack back to the cassette against the applied force. The vacuum motor as shown in Fig.3, which used to pick the currency from the cash cassettes is a $24 \mathrm{~V}$ DC stepper motor which will be operated using the Pulse Width Modulation signal.

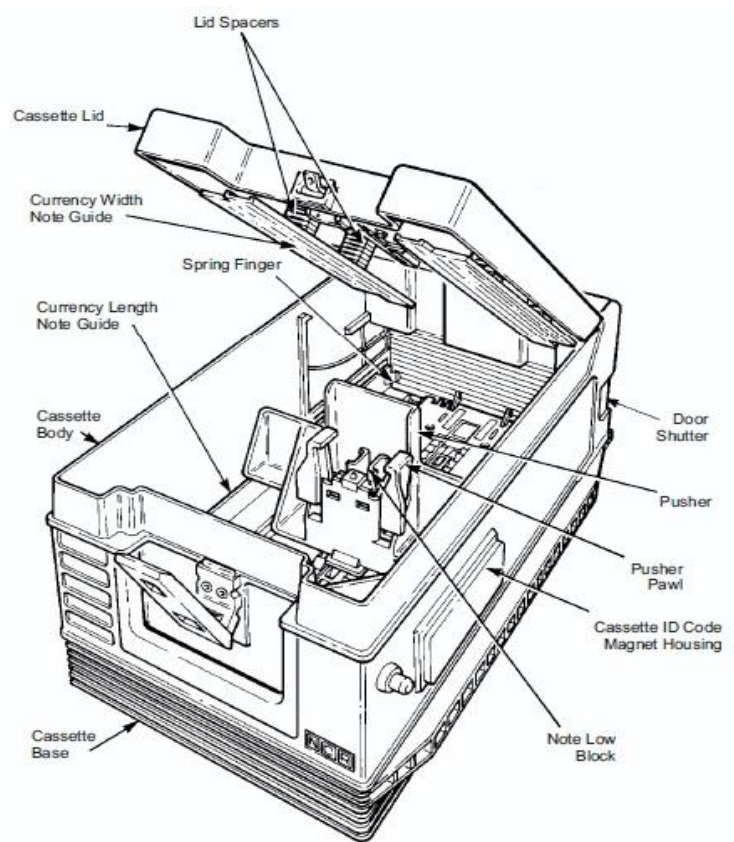

Fig.2 Cash cassette in dispenser unit

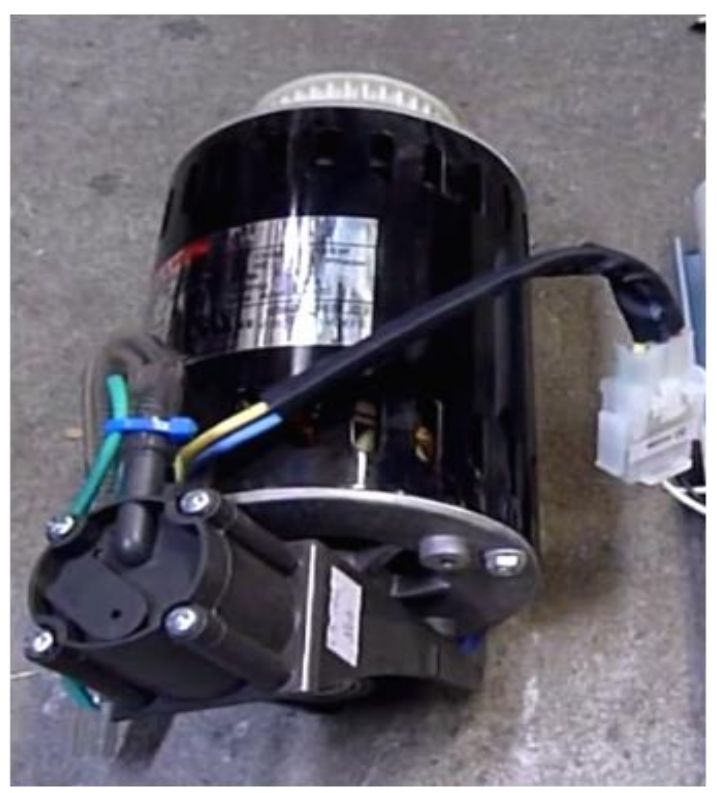

Fig.3 Vacuum motor

Difficulties in the vacuum motor based dispenser unit

- Unable to view or store pressure generated

- Incorrect number of cash withdrawal

- Manual operation 


\section{PROPOSED SYSTEM}

\subsection{Block Diagram of the Proposed System}

The vacuum motor present inside the Automated Teller Machine dispenser [9] is applied with power supply where the motor generates the vacuum which is been circulated within the hose pipe. The positive and negative terminal of the vacuum motor is linked to the relay circuit where one terminal of the hose pipe is given to the nozzle of the pressure flow sensor. Fig. 4 indicates the block diagram of the proposed system which uses ATMEGA-8 microcontroller unit to process the sensor values obtained from the pressure flow sensor and has been converted using Analog to Digital Converter (ADC). The pressure values obtained is displayed using a 16×2 LCD display.

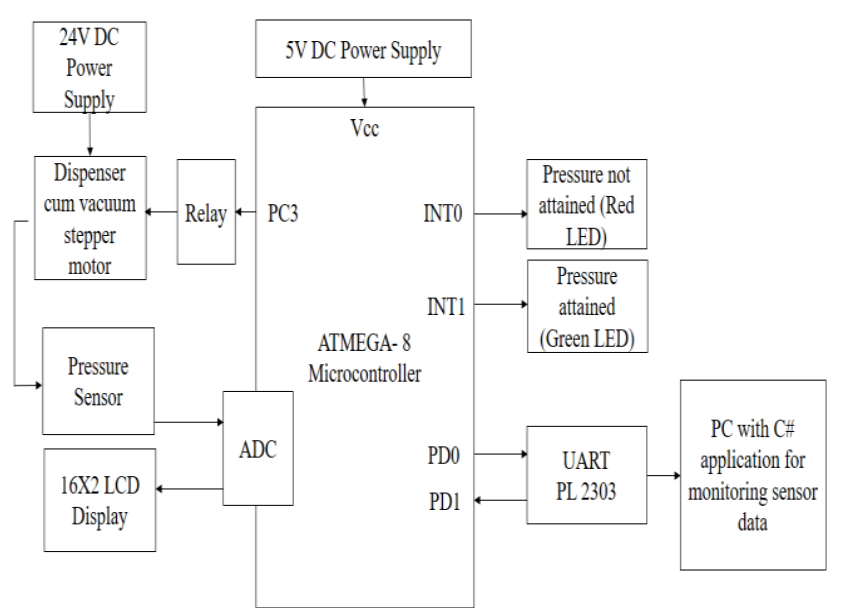

Fig.4 Block diagram of the proposed system

The pressure flow sensor contains a shaft inside, during the air flow through the sensor the shaft is been circulated thus the sensor sends the values to the microcontroller, in this system the threshold pressure value has been fixed as 850 millibar/sec hence the cut off pressure value has been set between $840-860$ millibar/sec.

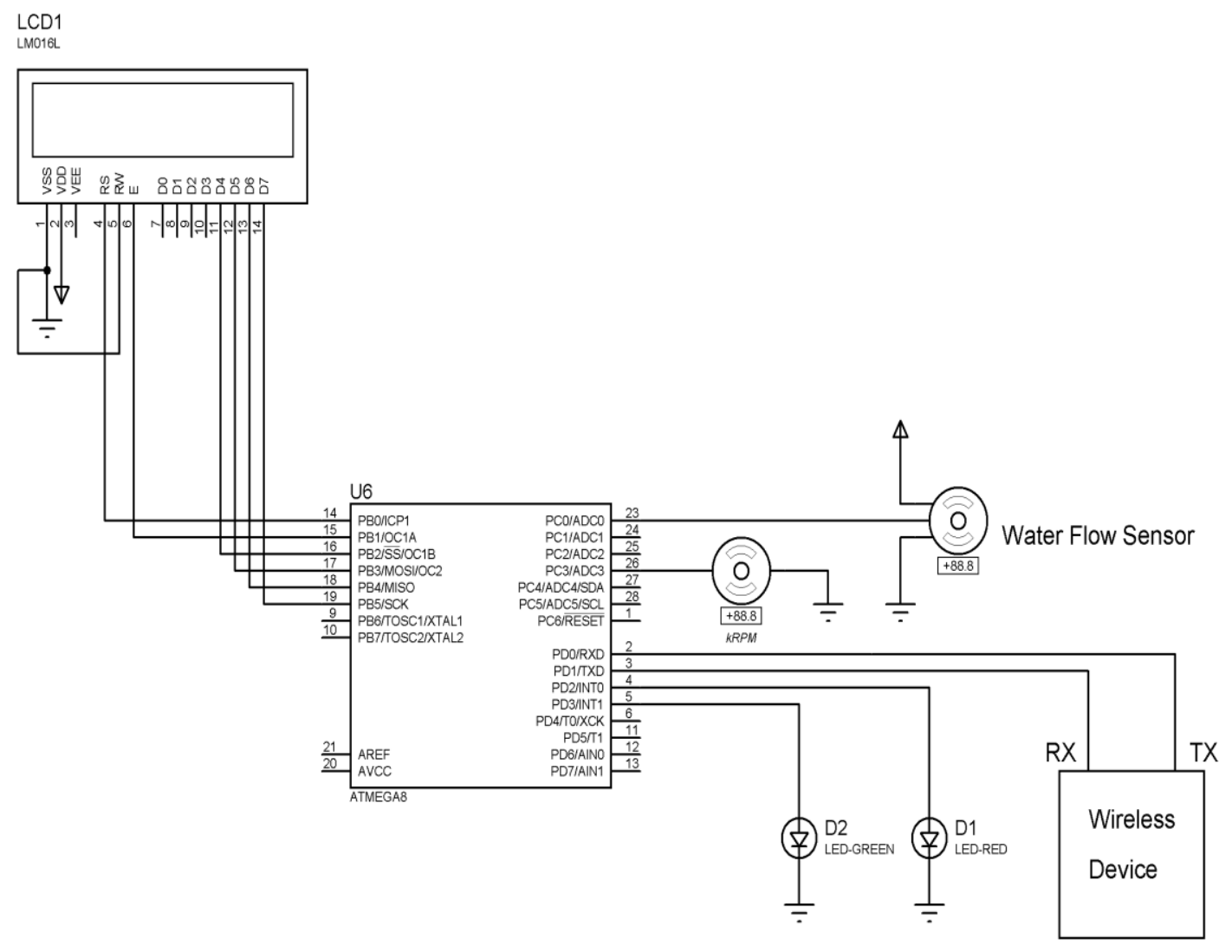

Fig.5 Circuit diagram of proposed diagram 


\subsection{Functional Operation of Proposed System}

Hardware part is designed using ATMEGA- 8 microcontroller, pressure flow sensor (YF-S201), LED setup to indicate the status of the motor, switch to control the motor, relay setup, LCD module and UART. The motor is turned on and input pressure is sensed by pressing the switch to control the motor in order to recognize the amount of pressure generated by the vacuum motor once the pressure is being applied through the flow sensor the pressure generated is viewed as output in the LCD display as shown in Fig.5, once the cut off pressure value is achieved the motor is set to turn off automatically. The pressure change can be exhibited in the personal computer using the datalogger software.

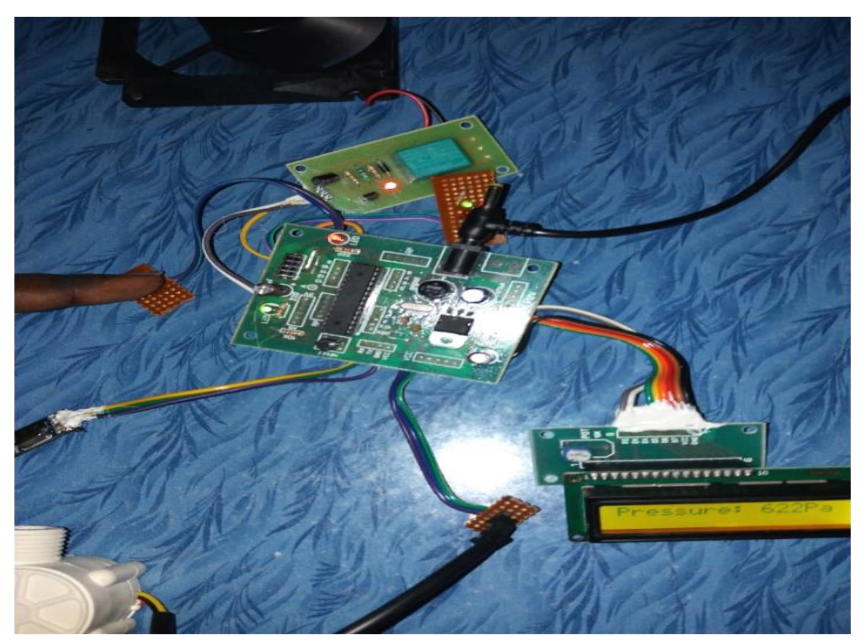

Fig.5: Sensing of pressure value using pressure sensor

\section{RESULTS}

Initially when the hardware setup is ON, the RESET button is pressed in the microcontroller the entire circuit gets reset and the motor attached to the controller starts working according to the instruction. After initialization the pressure can be applied from vacuum motor to the flow sensor and output is generated in the LCD and the datalogger software shown in Fig.6 and Fig.7 respectively.

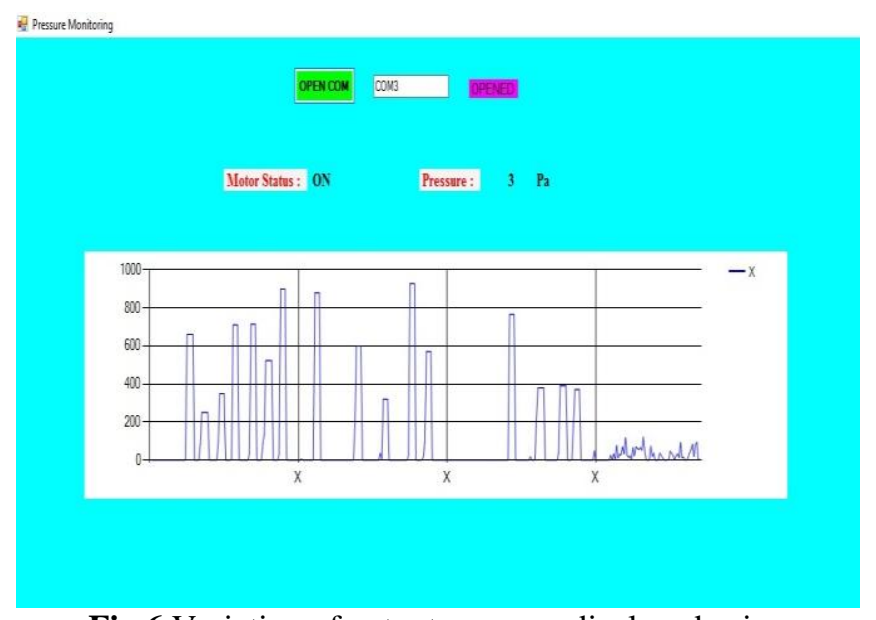

Fig.6 Variation of output pressure displayed using datalogger
Once the cut off pressure value is achieved the motor is switched off and the values are viewed in datalogger and the values retain till the exit of the datalogger software. The status of the motor is also being indicated in the datalogger.

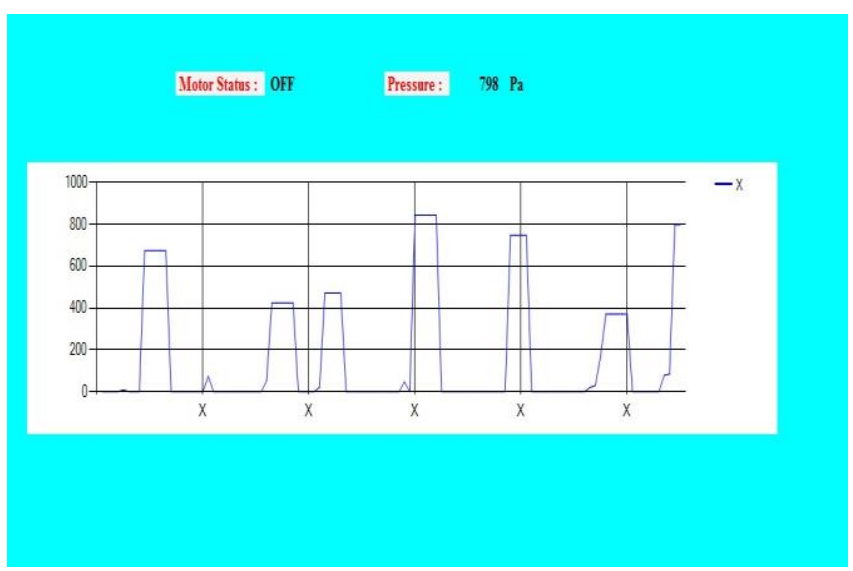

Fig.7 Output pressure data obtained

The graphical representation helps to notice the pressure variance and the status of the vacuum motor which could be stored for the future reference and to categorize the motor based on their performance and can be incorporated in appropriate dispenser unit.

\section{CONCLUSION}

Pressure measurement for vacuum motor in Automated Teller Machine dispenser provides easier detection of pressure generated by the vacuum motor. Screening of the pressure value and displaying the status of the motor is performed by interfacing the microcontroller with computer using UART serial communication. Also this system can be incorporated since it is small in size and easy to handle by the manufacturer. The only limitation is the datalogger software can store the details until the exit of the program. Thus this limitation can be overcome by enhancing the datalogger to save the data at the accomplishment of each process such that this values could be used for the future reference by the manufacturer.

\section{FUTURE ENHANCEMENT}

Future enhancement be able to be done by integrating hardware with the Internet of Things (IoT) to this project such that all the data acquired can be exhibited and stored in the ATM manufacturer web server, Currently, we have implemented system by considering only viewing of data plot for a single processing the future enhancement is extended by storing data for multiple processing.

\section{ACKNOWLEDGEMENT}

I thank Mrs. A. Vijayalakshmi, Associate Professor, ECE Department guiding me throughout the project and supporting me in giving technical ideas about the paper and motivating me to complete the work efficiently and successfully. 


\section{REFERENCES}

[1] Bernardo Ba'tiz-Lazo, Robert J.K Reid, "The Development of Cash- Dispensing Technology in the UK", IEEE Annals of the History of Computing, pp 32-45, 2011.

[2] Nuthan K, Nagarathna B M, SumanaNayaka R L, VidyaRathna B, "An Automated Teller Machine: Literature Review", International Journal of Novel Research in Computer Science and Software Engineering, Vol. 2, Issue 1,pp: (40-45), 2012.

[3] Dhiraj Vasant Kapare, Sadashiv Lkhande Savaji Kale, "Automatic Cash Deposite Machine with Currency Detection Using Fluorescent and UV Light" International Journal of Computational Engineering Research, Vol.3, Issue.3, 2011.

[4] Souvik Biswas, AnamitraBardhan Roy, Kishore Ghosh, NilanjanDev, "A Biometric Authentication Based Secured ATM Banking System”, International Journal of Advanced Research in Computer Science and Software Engineering, ISSN:2277 128X, Vol. 2, Issue 4, 2012.

[5] Pooja Mali ShrutiSalunkeRajashri, Mane Pooja Khatavkar, "Multilevel ATM Security Based On Two Factor Biometrics", International Journal of Engineering Research \& Technology (IJERT), Vol. 1, Issue 8, ISSN: 2278-0181, 2008.

[6] EssienEyo E, "Security proposed for Automated Teller Machine”, Elixir Adoc Network ISSN: 31643167, 2011.

[7] Anurag AnandDuvey, Dinesh Goyal, Dr. Naveen Hemrajani, "A Reliable ATM Protocol and Comparative Analysis on Various Parameters with Other ATM Protocols", International Journal of Communication and Computer Technologies, ISSN: 2278-9723, 2013.

[8] Akashchoubey, Amarjot Singh, SrikrishnaKaranam, Devinder Kumar, and KetanBacchuwar, "A Novel Signature Verification Based Automatic Teller Machine", International Journal of Information and Electronics Engineering, Vol. 2, pp 198-207, 2009.

[9] https://www.ncr.com/financial-services/cashdispenser-atms

[10] http://www.oki.com/en/130column/12.html

\section{BIOGRAPHIES}

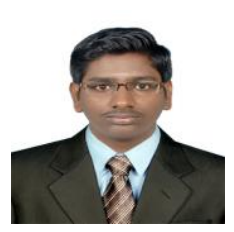

Mr. S.R Jayalogainiyan is now pursuing his Master of Technology (M.Tech) in VLSI \& Embedded Systems at Sri Manakula Vinayagar Engineering College,Puducherry, India

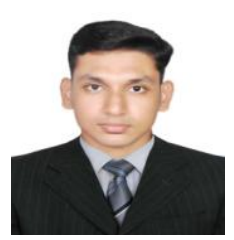

Mr. K.S Srijan is now pursuing his Master of Technology (M.Tech) in VLSI \& Embedded Systems at Sri Manakula Vinayagar Engineering College, Puducherry, India.

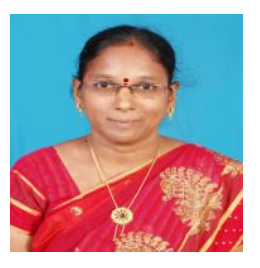

Mrs. A. Vijayalakshmi completed her B.E in ECE from Madras University and M.E in ECE from Pondicherry University. She is now working as Associate Professor in ECE Department at Sri Manakula Vinayagar Engineering College, Puducherry, India. She has over 18 years of teaching experience. 